\title{
THE UTILITY OF WHOLE EXOME SEQUENCING IN DIAGNOSING PEDIATRIC NEUROLOGICAL DISORDERS
}

\author{
Muthaffar OY*
}

*Corresponding Author: Osama Y. Muthaffar, M.D., Department of Pediatrics, King Abdulaziz University, Jeddah, PO Box 80215, Jeddah 21589, Kingdom of Saudi Arabia. Tel.: +96-12-640-1000 (ext. 20208). Fax: 996-12-640-3975. E-mail: oymuthaffar@kau.edu.sa; osamam@hotmail.com

\begin{abstract}
Pediatric neurological disorders have a wide spectrum of clinical presentations and can be challenging to diagnose. Whole exome sequencing (WES) is increasingly becoming an integral diagnostic tool in medicine. It is cost-effective and has high diagnostic yield, especially in consanguineous populations. This study aims to review WES results and its value in diagnosing neurological disorders. A retrospective chart review was performed for WES results between the period of January 2018 to November 2019. Whole exome sequencing was requested for children with unexplained neurological signs and symptoms such as epilepsy, developmental delay, visual impairment, spasticity, hypotonia and magnetic resonance imaging (MRI) brain changes. It was conducted for children in a pediatric neurology clinic of a tertiary center at Jeddah, Saudi Arabia. Twenty-six children with undiagnosed neurological conditions were identified and underwent WES diagnosis. Nineteen patients $(73.0 \%)$ of the cohort were diagnosed with pathogenic variants, likely pathogenic variants or variants of unknown significance (VUS). Consanguinity was positive in 18 families of the cohort $(69.0 \%)$. Seven patients showed homozygous mutations. Five patients had heterozygous mutations. There were six patients with VUS and six patients had negative WES results. Whole exome sequencing showed a high diagnostic rate in this group of children with variable neurological disorders.
\end{abstract}

Keywords: Consanguinity; Developmental delay; Neurological disorders; Saudi Arabia; Whole exome sequencing (WES).

Department of Pediatrics, King Abdulaziz University, Jeddah, Kingdom of Saudi Arabia

\section{INTRODUCTION}

Childhood neurological disorders are a vast group of heterogenous conditions with a myriad of clinical presentations. Reaching a diagnosis can be challenging, time-consuming and costly. Sometimes, multiple imaging, laboratory investigations and ancillary procedures are exhausted trying to reach a final diagnosis [1]. Families also suffer from the dilemma of multiple investigations. Many neurological conditions in children present with variable degrees of presentations as well as different stages of progression, thus, multiple metabolic and genetic investigations are requested. Recently, whole exome sequencing (WES) has become an important diagnostic tool for many presumed genetic or idiopathic neurological conditions [2]. Whole exome sequencing is becoming rapidly available, costeffective and can be a shortcut to the diagnosis. Reaching a diagnosis in a child with a neurological disorder, counseling parents and preventing recurrences of similar conditions in his/her family is the essence of pediatric neurology practice. Nowadays, phenotype-based genetic testing and panels are slowly being substituted by WES [3]. The depth of screening using WES and variant coverage as well as identification of novel and pathogenic variants is rapidly improving. The increasing rate of WES sensitivity, expanding genetic databases, shorter turn-around time and decreasing prices of WES are appealing and promising [4].

In the Middle East region and Africa, consanguinity is common. In Saudi Arabia, 52.0-67.0\% of marriages are from the same family and tribe $[5,6]$. This cultural practice can be associated with a higher percentage of neurogenetic and metabolic conditions [7]. The diagnostic yield of WES can range from 20.0 to $70.0 \%$ with a higher yield in consanguineous populations [8-12]. Few studies in the Middle East reviewed the outcomes of WES in children with neurological disorders in a population with 
high consanguinity rate such as Saudi Arabia [13-16]. Such characteristics could increase the yield of WES. In this cohort, the clinical characteristics and WES results of children with variable neurological disorders in Saudi Arabia are reviewed.

\section{MATERIAL AND METHODS}

A retrospective chart review for 26 children with undiagnosed neurological conditions was performed from January 2018 to November 2019. These neurological disorders ranged between developmental delay, hypotonia, epilepsy, loss of vision, ataxia, white matter changes, intellectual delay, encephalopathy and metabolic abnormalities. This review was conducted for children attending the pediatric neurology clinic at King Abdulaziz University, Jeddah, Saudi Arabia. Medical history, patients' demographics, history of consanguinity (second cousin or closer), clinical examination and neuroimaging were reviewed and documented. All patients underwent proband-only WES. Further segregation analyses were performed for parents planning future pregnancies. Whole exome sequencing was performed in an accredited laboratories, Centogene in Rostock, Germany and Blueprint laboratories in Helsinki, Finland. Variant classifications into five classes were based on American College of Medical Genetics and Genomics (ACMG) guidelines [17] (Table 1). All patients signed a consent form for WES. The study was approved by the King Abdulaziz University Research Ethics Committee.

\section{RESULTS}

There were 19 males and seven females included in the study. Age of presentation ranged from 1 to 12 years (mean 4.8 years). Nineteen patients had positive WES results $(73.0 \%)$, five patients with variants of unknown significance (VUS) that matches the clinical picture, one patient with VUS that does not explain the observed clinical characteristics. The remaining six patients were negative. The average age in the WES-positive group was 4.9 years and the average age in the WES-negative group was 4.5 years. Consanguinity was positive in $69.0 \%$ of the cohort, most of them were in the WES-positive group (Table 2).

The WES-positive cohort results were classified into pathogenic, likely pathogenic variants and VUS. In the homozygous and heterozygous groups, pathogenic and likely pathogenic mutations were found. These mutations, when paired with the phenotype, confirms the clinical picture (Tables 3 and 4). The VUS group was mainly clinically upgraded to clinically significant variants as those patients have matched genotype and phenotype. No benign or likely benign variants were detected in this cohort.
Table 1. American College of Medical Genetics and Genomics (ACMG) classification of variants [17].

\begin{tabular}{|l|l|}
\hline Class 1 & Pathogenic \\
\hline Class 2 & Likely pathogenic \\
\hline Class 3 & Variant of uncertain significance (VUS) \\
\hline Class 4 & Likely benign \\
\hline Class 5 & Benign \\
\hline
\end{tabular}

Table 2. Cohort demographics: WES positive and negative.

\begin{tabular}{|l|l|l|}
\hline & \multicolumn{1}{|c|}{$\begin{array}{c}\text { Positive } \\
\boldsymbol{n}(\mathbf{\%})\end{array}$} & \multicolumn{1}{|c|}{$\begin{array}{c}\text { Negative } \\
\boldsymbol{n}(\mathbf{\%})\end{array}$} \\
\hline Males & 14 & 5 \\
\hline Females & 6 & 1 \\
\hline Total & $20(77.0)^{\mathrm{a}}$ & $6(23.0)$ \\
\hline Age (mean) & 4.9 years & 4.5 years \\
\hline Consanguinity & $14(53.0)$ & $4(15.0)$ \\
\hline
\end{tabular}

a One of the 20 patients had positive WES results but does not explain the phenotype (patient 15 in Table 3).

Eight patients had homozygous mutations, six patients had heterozygous mutations and six patients were carrying VUS. All patients had variable neurological presentations such as: epilepsy, intellectual delay, motor delay, regression of milestones, hypotonia, visual abnormalities, ataxia and MRI brain changes. Similar family history in another sibling or relative was reported in seven patients with positive WES result.

One patient had ITGA7 classified as VUS that does not explain the phenotype. He was developmentally normal then developed regression of milestones and a middle cerebral artery stroke after meningoencephalitis. An ITGA7 mutation was reported to be associated with congenital muscular dystrophy, neonatal hypotonia, proximal atrophy and scoliosis, which were not apparent in this patient before his regression.

Variants of unknown significance with consistent phenotypes were seen in five patients (Table 3). The MLC1 mutation in patient \#16 was consistent with megalencephalic leukoencephalopathy with subcortical cysts type 1 . The patient developed ataxia and convulsions. Brain MRI also showed expected white matter changes and temporal cysts. Patient \#17 had a SLC6A3 mutation compatible with infantile Parkinsonism-dystonia type 1 . He presented with orolingual, upper and lower limbs dystonia and was initially diagnosed as cerebral palsy. Patient \#18 was diagnosed with intractable infantile spasms and failed to respond to multiple antiseizure medications for several months. He also developed regression of milestones. A PNPO mutation 
Table 3. Homozyogous, heterozygous and variants of uncertain significance VUS groups.

\begin{tabular}{|c|c|c|c|c|c|c|c|c|}
\hline \multicolumn{9}{|c|}{ Homozygous Mutations } \\
\hline \# & $\begin{array}{l}\text { Sex- } \\
\text { Age }\end{array}$ & $\begin{array}{l}\text { Family } \\
\text { History }\end{array}$ & $\begin{array}{l}\text { Consan- } \\
\text { guinity }\end{array}$ & Clinical Characteristics & Onset & Genes & Variant & Diagnoses \\
\hline 1 & F-6 & yes & yes & $\begin{array}{l}\text { motor delay; hypotonia; } \\
\text { scoliosis; respiratory difficulties } \\
\text { in neonatal period; normal } \\
\text { congnitions, areflexia }\end{array}$ & 1 year & PIEZO2 & $\begin{array}{l}\text { pathogenic: } \\
\text { c.273_279del, } \\
\text { p.(Pro92Thrfs*18) }\end{array}$ & $\begin{array}{l}\text { piezo-type } \\
\text { mechanosensitive ion } \\
\text { channel component; } \\
\text { OMIM: } 613629\end{array}$ \\
\hline 2 & M-1 & no & yes & $\begin{array}{l}\text { severe hypotonia; reduced tendon } \\
\text { reflexes; motor/speech delay; } \\
\text { cerebellar atrophy; cerebellar } \\
\text { cyst; elevated serum CPK }\end{array}$ & birth & $F K R P$ & $\begin{array}{l}\text { likely pathogenic: } \\
\text { c.204del, } \\
\text { p.(Ser69Profs*60) }\end{array}$ & $\begin{array}{l}\text { MDDGA5 (congenital with } \\
\text { brain/eye anomalies), type } \\
\text { A5 (MDDGA5); OMIM: } \\
613153\end{array}$ \\
\hline 3 & M-2 & no & yes & $\begin{array}{l}\text { vision loss; nystagmus; severe } \\
\text { retinal dysfunction }\end{array}$ & 2 months & RPGRIPI & \begin{tabular}{|l|} 
pathogenic: \\
c.1107del, \\
p.(Glu370Asnfs $* 5)$ \\
\end{tabular} & $\begin{array}{l}\text { Leber congenital amaurosis } \\
\text { type 6, OMIM: } 613826\end{array}$ \\
\hline 4 & M-7 & no & yes & $\begin{array}{l}\text { intractable epilepsy; global } \\
\text { developmental delay; poor } \\
\text { vision }\end{array}$ & 3 years & TPP1 & $\begin{array}{l}\text { pathogenic: } \\
\text { c.616C }>\text { T, } \\
\text { p.(Arg206Cys }) \\
\end{array}$ & $\begin{array}{l}\text { neuronal ceroid } \\
\text { lipofuscinosis type } 2 \text {, } \\
\text { OMIM: } 204500\end{array}$ \\
\hline 5 & M-8 & no & yes & $\begin{array}{l}\text { poor hearing; encephalopathy; } \\
\text { MRI: white matter changes }\end{array}$ & 4 years & $B T D$ & $\begin{array}{l}\text { pathogenic: } \\
\text { c.1618C }>\mathrm{T}, \\
\text { p.(Arg540Cys) }\end{array}$ & biotinidase deficiency \\
\hline 6 & M-6 & yes & yes & $\begin{array}{l}\text { ataxia; delayed motor } \\
\text { milestones; mild intellectual } \\
\text { delay; MRI: cerebellar atrophy }\end{array}$ & 1 year & SPTBN2 & $\begin{array}{l}\text { likely pathogenic: } \\
\text { c.6258_6261delGAGA, } \\
\text { p.(Lys2088Glyfs*228) }\end{array}$ & $\begin{array}{l}\text { infantile-onset } \\
\text { spinocerebellar ataxia } \\
\text { type 5 }\end{array}$ \\
\hline 7 & F-8 & yes & yes & $\begin{array}{l}\text { ataxia; delayed motor } \\
\text { milestones; mild intellectual } \\
\text { delay; MRI: cerebellar atrophy }\end{array}$ & 1 year & SPTBN2 & $\begin{array}{l}\text { likely pathogenic: } \\
\text { c.6258_6261delGAGA, } \\
\text { p.(Lys2088Glyfs*228) }\end{array}$ & \begin{tabular}{|l|} 
infantile-onset \\
spinocerebellar ataxia \\
type 5
\end{tabular} \\
\hline 8 & M-9 & yes & yes & $\begin{array}{l}\text { ataxia; oculomotor apraxia; } \\
\text { telangiectasia; MRI: cerebellar } \\
\text { atrophy }\end{array}$ & 3 years & $A T M$ & $\begin{array}{l}\text { likely pathogenic: } \\
\text { c.9066del, } \\
\text { p.(Gly3023Alafs*10) }\end{array}$ & ataxia telangiectasis \\
\hline \multicolumn{9}{|c|}{ Heterozygous Mutations } \\
\hline 9 & F-7 & no & no & $\begin{array}{l}\text { delayed language/motor } \\
\text { development; intellectual } \\
\text { disability; hypotonia; } \\
\text { generalized seizures; infantile } \\
\text { spasms; visual impairment; } \\
\text { normal MRI; normal } \\
\text { metabolic profile }\end{array}$ & 1 year & NTRK2 & \begin{tabular}{|l|} 
pathogenic: \\
c.1301A $>\mathrm{G}$, \\
p.(Tyr434Cys)
\end{tabular} & $\begin{array}{l}\text { early infantile epilectic } \\
\text { encephalopathy type } 58, \\
\text { OMIM: } 617830\end{array}$ \\
\hline 10 & M-3 & yes & no & $\begin{array}{l}\text { acute necrotizing } \\
\text { encephalopathy; generalized } \\
\text { seizures; spasticity; coma and } \\
\text { death; brain MRI: symmetric } \\
\text { thalamic hyperintense lesions }\end{array}$ & 3 years & $R A N B P 2$ & $\begin{array}{l}\text { pathogenic: } \\
\text { c.1754C }>\text { T, } \\
\text { p.(Thr585Met) }\end{array}$ & $\begin{array}{l}\text { acute infection-induced } \\
\text { encephalopathy-type } 3 \text {, } \\
\text { OMIM: } 608033\end{array}$ \\
\hline 11 & M-4 & no & no & $\begin{array}{l}\text { developmental delay; neonatal } \\
\text { hypotonia; autistic-like } \\
\text { behavior; epilepsy }\end{array}$ & 1 year & SHANK3 & $\begin{array}{l}\text { likely pathogenic: } \\
\text { c. } 2313+1 \mathrm{G}>\mathrm{A}\end{array}$ & $\begin{array}{l}\text { Phelan-McDermid } \\
\text { syndrome, OMIM: } 606232\end{array}$ \\
\hline 12 & M-9 & no & no & $\begin{array}{l}\text { ataxia; ADHD; delayed speech/ } \\
\text { language development; motor } \\
\text { delay; hypotonia; normal EEG } \\
\text { and brain MRI }\end{array}$ & 2 years & KAT6A & $\begin{array}{l}\text { likely pathogenic: } \\
\text { c. } 1483-1 \mathrm{G}>\mathrm{A}\end{array}$ & $\begin{array}{l}\text { mental retardation type } 32 \text {, } \\
\text { OMIM: } 616268\end{array}$ \\
\hline 13 & M-1 & no & no & $\begin{array}{l}\text { intractable neonatal seizures; } \\
\text { normal brain MRI }\end{array}$ & 1 month & PACS2 & $\begin{array}{l}\text { pathogenic: } \\
\text { c.625G }>\text { A, } \\
\text { p.(Glu209Lys) }\end{array}$ & $\begin{array}{l}\text { early infantile epileptic } \\
\text { encephalopathy type } 66 \text {, } \\
\text { OMIM: } 618067\end{array}$ \\
\hline 14 & F-4 & no & yes & $\begin{array}{l}\text { intractable focal seizures; } \\
\text { normal brain MRI }\end{array}$ & 3 months & SCN1A & $\begin{array}{l}\text { likely pathogenic: } \\
\text { c.1377G }>\text { C, } \\
\text { p.(Gln459His) }\end{array}$ & $\begin{array}{l}\text { early infantile epileptic } \\
\text { encephalopathy type } 6 \\
\text { (Dravet syndrom), OMIM: } \\
607208\end{array}$ \\
\hline
\end{tabular}

Variant(s) of Uncertain Significance

\begin{tabular}{|l|l|l|l|l|l|l|l|}
\hline 15 & M-6 & no & yes & $\begin{array}{l}\text { MCA stroke; dystonia; } \\
\text { spasticity; regression of } \\
\text { milestones; delayed language/ } \\
\text { motor development; focal } \\
\text { seizures and abnormal brain } \\
\text { myelination on MRI }\end{array}$ & 4 years & ITGA7 & $\begin{array}{l}\text { c.1601C>T, } \\
\text { p.(Ala534Val) }\end{array}$ \\
AR & $\begin{array}{l}\text { congenital muscular } \\
\text { dystrophy/hypotonia, } \\
\text { OMIM: 613204 }\end{array}$ \\
\hline
\end{tabular}


Continuation of the previous page

\begin{tabular}{|c|c|c|c|c|c|c|c|c|}
\hline 16 & F-5 & no & yes & $\begin{array}{l}\text { ataxia; frequent falls; } \\
\text { macrocephaly, epilepsy and } \\
\text { ADHD; MRI: megalencephalic } \\
\text { leukoencephalopathy with } \\
\text { subcortical cysts }\end{array}$ & 2 years & $\begin{array}{l}M L C 1 \\
\text { AR }\end{array}$ & $\begin{array}{l}\text { c. } 275 \mathrm{C}>\mathrm{A}, \\
\text { p.(Pro92His) }\end{array}$ & $\begin{array}{l}\text { megalencephalic } \\
\text { leukoencephalopathy with } \\
\text { subcortical cysts type } 1, \\
\text { OMIM: } 604004\end{array}$ \\
\hline 17 & M-3 & no & yes & $\begin{array}{l}\text { delayed speech/language } \\
\text { development; dyskinesia; } \\
\text { dystonia; infantile onset of the } \\
\text { disease; paroxysmal dystonia; } \\
\text { MRI: brain atrophy }\end{array}$ & 2 years & $\begin{array}{l}S L C 6 A 3 \\
\text { AR }\end{array}$ & $\begin{array}{l}\text { c. } 851 \mathrm{G}>\mathrm{A}, \\
\text { p.(Gly284Glu) }\end{array}$ & $\begin{array}{l}\text { infantile Parkinsonism } \\
\text { dystonia type } 1, \text { DTDS, } \\
\text { PMID: } 21777827\end{array}$ \\
\hline 18 & M-1 & yes & yes & intractable infantile spasms & 3 months & $\begin{array}{l}P N P O \\
\text { AR }\end{array}$ & $\begin{array}{l}\text { c. } 256 \mathrm{~T}>\mathrm{C}, \\
\text { p.(Cys86Arg) }\end{array}$ & PNPO, OMIM: 603287 \\
\hline 19 & F-6 & yes & yes & $\begin{array}{l}\text { ataxia and oculomotor apraxia; } \\
\text { brain MRI: molar tooth sign }\end{array}$ & 2 years & $\begin{array}{l}C C 2 D 2 A \\
\text { AR }\end{array}$ & $\begin{array}{l}\text { c.916_927del, } \\
\text { p.(Pro306_Leu309del) }\end{array}$ & $\begin{array}{l}\text { Joubert syndrome type } 9 \text {, } \\
\text { OMIM: } 612285\end{array}$ \\
\hline 20 & M-2 & no & yes & $\begin{array}{l}\text { fair hair; global developmental } \\
\text { delay; hearing impairment; } \\
\text { infantile onset of the disease; } \\
\text { motor delay; muscular } \\
\text { hypotonia; visual impairment; } \\
\text { focal epilepsy; MRI: brain } \\
\text { atrophy }\end{array}$ & 3 months & \begin{tabular}{|l|} 
SPATA5 \\
AR \\
and \\
TIMMDC1
\end{tabular} & $\begin{array}{l}\text { c.1058A }>\text { T, } \\
\text { p.(Asp353Val) } \\
\text { and } \\
\text { c.230T>C, p.Ile77Thr) }\end{array}$ & $\begin{array}{l}\text { EHLMRS, OMIM: } 616577 \\
\text { mitochondrial complex I } \\
\text { deficiency, OMIM: } 618251\end{array}$ \\
\hline
\end{tabular}

\#: patient number; F: female; M: male; OMIM: Online Mendelian Inheritance in Man; CPK: creatine phosphokinase; MDDGA5: muscular dystrophydystroglycanopathy type A5; MRI: magnetic resonance imaging; ADHD: attention deficit hyperactivity disorder; EEG: electroencephalogram; MCA: middle cerebral artery; DTDS: dopamine transporter deficiency syndrome (DTDS); PMID: PubMed reference number; AR: autosomal recessive; PNPO: pyridoxamine 5'-phosphate oxidase; EHLMRS: epilepsy, hearing loss and mental retardation syndrome.

a Autosomal mitochondrial.

Table 4. Whole exome sequencing negative group.

\begin{tabular}{|l|l|c|c|l|l|}
\hline \# & Sex-Age & $\begin{array}{c}\text { Family } \\
\text { History }\end{array}$ & $\begin{array}{c}\text { Consan- } \\
\text { guinity }\end{array}$ & Clinical Characteristics & Onset \\
\hline 1 & F-1 & no & yes & epilepsy and developmental delay; brain MRI: normal & 4 months \\
\hline 2 & M-7 & no & yes & epilepsy and developmental delay; brain MRI: normal & 2 years \\
\hline 3 & M-2 & no & no & intractable epilepsy; poor vision and global developmental delay; brain MRI: normal \\
\hline 4 & M-12 & no & yes & global developmental delay; brain MRI: basal ganglia enhancement; metabolic work-up: negative & 6 years \\
\hline 5 & M-4 & yes & yes & intractable focal epilepsy and ADHD & 1 year \\
\hline 6 & M-2 & no & yes & microcephaly; motor delay; brain MRI: white matter changes & 6 months \\
\hline
\end{tabular}

MRI: magnetic resonance imaging; ADHD: attention deficit hyperactivity disorder.

was reported once. He was put on pyridoxal 5 phosphate cofactor therapy. No more seizures were reported by the parents and he is now developmentally up to age after 1 year of follow-up. Targeted analysis of both parents showed heterozygous mutations in both (Figure 1). Patient \#19 was found to have ataxia and oculomotor apraxia. Brain MRI showed classic molar tooth sign. The $C C 2 D 2 A$ mutation is associated with Joubert syndrome. Patient \#20 had infantile onset of developmental delay, hearing impairment, hypotonia, visual impairment, focal epilepsy, high serum lactic acid and brain MRI showed brain atrophy. Mutations in SPATA 5 and TIMMDC1 were reported to cause epilepsy, hearing loss, and mental retardation syndrome and mitochondrial complex I deficiency, respectively.

\section{DISCUSSION}

The introduction of WES in medicine has changed the way of physician's approach to patients. The number of newly diagnosed neurogenetic conditions and mutations are increasing.

Multiple studies worldwide reviewed WES findings, however, few in the Middle East region. In Saudi Arabia, the Arabic ethnic background is the main population structure. Consanguinity is common in marriages. Thus, more metabolic and neurogenetic conditions are prevalent in our region.

In this study, 19 patients $(73.0 \%)$ out of 26 patients had genetically and phenotypically consistent findings. In the WES-positive group, consanguinity was present in 

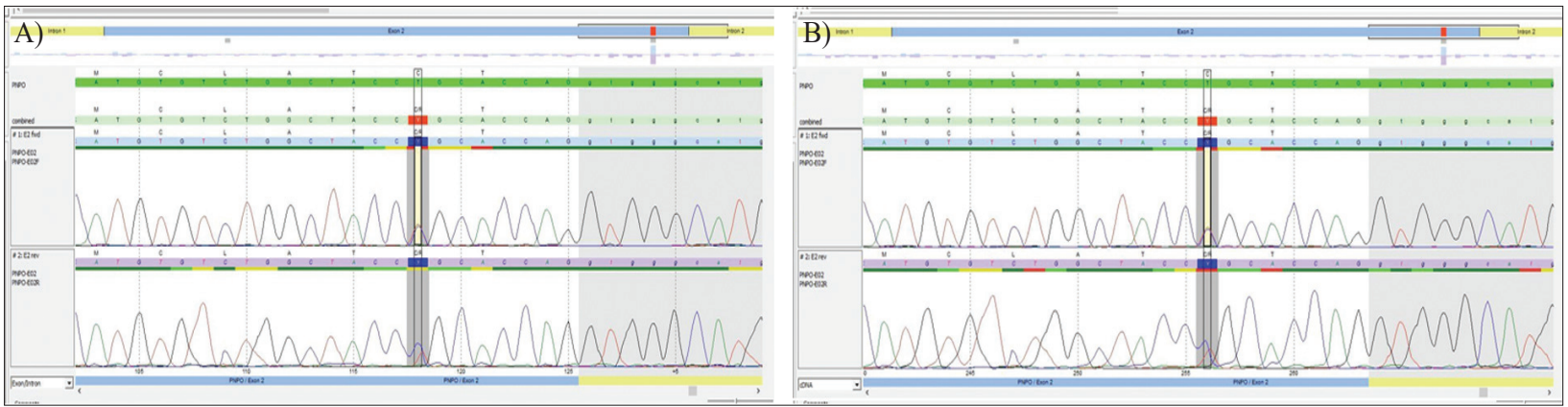

Figure 1. Parents of a child with an inherited $P N P O$ gene mutation. They are cousins and both are heterozygous. A) Mother; B) father.
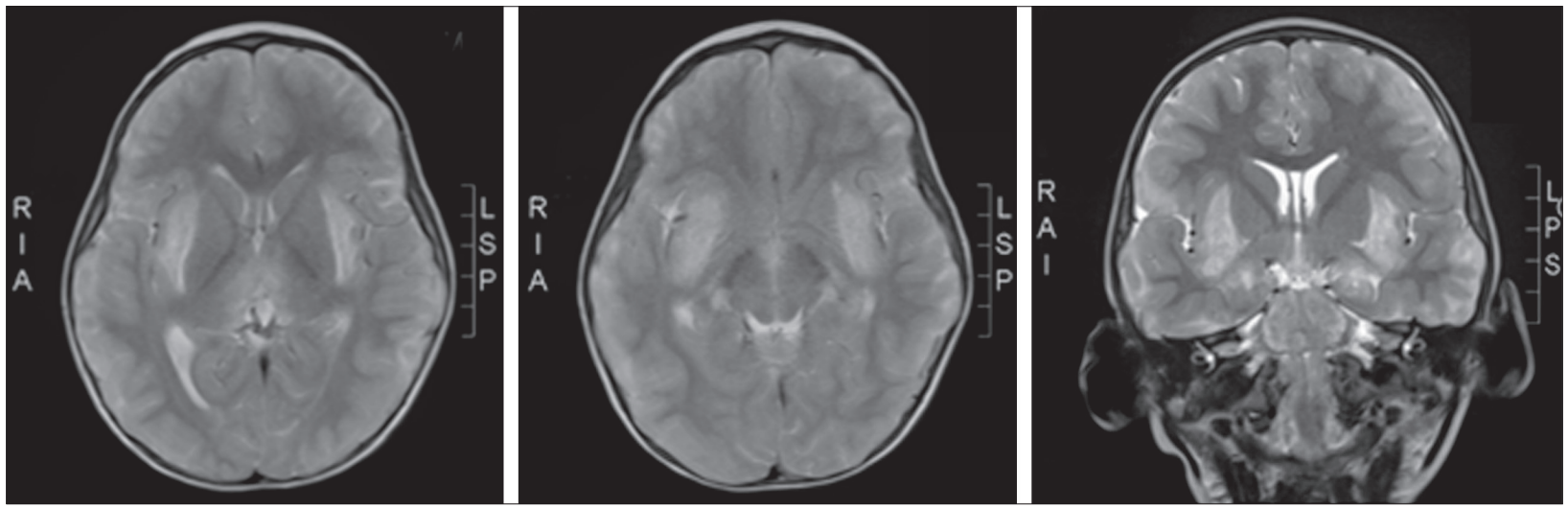

Figure 2. Brain MRI in a child with acute necrotizing encephalopathy and a positive RANBP2 gene mutation. Brain MRI showing increased $\mathrm{T} 2$ hyperintensity of basal ganglia (A, B, and C).

$53.0 \%$ of the families whereas in the WES-negative it was $15.0 \%$. Compared to other studies in the region $[18,19]$ $43.0-49.0 \%$ of WES results showed clinically significant results in Saudi Arabia. Other studies from the United Arab Emirates and Qatar, reached 54.0-68.0\% genetically confirmed diagnoses $[14,15]$. Larger studies including 2000-3000 WES samples from different ethnic regions, showed a lower diagnostic rate, around $25.0 \%$ from the USA [11], Care4Rare Canada [20] and Finding of Rare Disease Genes (FORGE) [21]. Consanguinity was positive in 18 families of the cohort $(69.0 \%)$. In other studies, a similar rate of $66.0-75.0 \%$ of consanguinity was reported $[12,14]$. The most common features of WES results in the Middle East are a high percentage of consanguinity and a higher positivity rate of WES results [22,23].

Whole exome sequencing has a direct impact on management [24]. For example, patient number \#5 (Table 3) had a positive $B T D$ mutation classified as pathogenic. He was 7 years old when diagnosed with biotinidase deficiency. His symptoms were noticed by his parents since he was 3 years old. He had hearing problems, ataxia, convulsions and intermittent encephalopathy. He was in coma and ventilatory-dependent when WES was requested. Once started on biotin supplements, he gradually started to improve. Currently, he is off the ventilator. He is redeveloping motor gains with physical therapy. He has not experienced any more convulsions and his hearing has improved.

Another patient in the cohort has a pathogenic $R A N$ $B P 2$ gene mutation. He presented with acute necrotizing encephalopathy (ANE) (Figure 2). A few months after being diagnosed, his sister was also diagnosed with clinical ANE. Unfortunately, both siblings died despite aggressive immunotherapy.

In conclusion, WES is an integral diagnostic tool in a pediatric neurology clinic. It is of great importance to unravel the diagnostic odyssey of many neurological and neurogenetic conditions. Family counseling, prevention of recurrence and treatment depends on proper genetic diagnoses. Pricing of WES is still a challenge at many centers and countries $[25,26]$. Sometimes, WES results can also take more than 2-3 months that can delay WES-focused medical care.

The high yield of WES results in this study, though a small number of patients, is compatible with similar studies. This study promotes performing WES in childhood neurological disorders, especially when these is a similar family history and positive consanguinity.

Declaration of Interest. The authors report no conflicts of interest. The authors alone are responsible for the content and writing of this article. 


\section{REFERENCES}

1. van Nimwegen KJM, Schieving JH, Willemsen MAA, Veltman JA, van der Burg S, van der Wilt GJ, et al. The diagnostic pathway in complex paediatric neurology: A cost analysis. Eur J Paediatr Neurol. 2015; 19(2): 233-239.

2. Fogel BL, Satya-Murti S, Cohen BH. Clinical exome sequencing in neurologic disease. Neurol Clin Pract. 2016; 6(2): 164-176.

3. Kong S, Lee I, Liu X, Hirschhorn JN, Mandl KD. Measuring coverage and accuracy of whole-exome sequencing in clinical context. Genet Med. 2018; 20(12): 1617-1626.

4. Ng SB, Buckingham KJ, Lee C, Bigham AW, Tabor HK, Dent KM, et al., Exome sequencing identifies the cause of a mendelian disorder. Nat Genet. 2010; 42(1): 30-35.

5. el-Hazmi MA, al-Swailem AR, Warsy AS, al-Swailem AM, Sulaimani R, al-Meshari AA. Consanguinity among the Saudi Arabian population. J Med Genet. 1995; 32(8): 623-626.

6. al Husain M, al Bunyan M. Consanguineous marriages in a Saudi population and the effect of inbreeding on prenatal and postnatal mortality. Ann Trop Paediatr. 1997; 17(2): 155-160.

7. Kahrizi K, Hu H, Hosseini M, Kalscheuer VM, Fattahi Z, Beheshtian M, et al. Effect of inbreeding on intellectual disability revisited by trio sequencing. Clin Genet. 2019; 95(1): 151-159.

8. Alazami AM, Patel N, Shamseldin HE, Anazi S, AlDosari MS, Alzahrani F, et al. Accelerating novel candidate gene discovery in neurogenetic disorders via whole-exome sequencing of prescreened multiplex consanguineous families. Cell Rep. 2015; 10(2): 148-161.

9. Yang Y, Muzny DM, Reid JG, Bainbridge MN, Willis A, Ward PA, et al. Clinical whole-exome sequencing for the diagnosis of Mendelian disorders. N Engl J Med. 2013; 369(16): 1502-1511.

10. Lee H, Deignan JL, Dorrani N, Strom SP, Kantarci S, Quintero-Rivera F, et al. Clinical exome sequencing for genetic identification of rare Mendelian disorders. JAMA. 2014; 312(18): 1880-1887.

11. Yang Y, Muzny DM, Xia F, Niu Z, Person R, Ding $\mathrm{Y}$, et al. Molecular findings among patients referred for clinical whole-exome sequencing. JAMA. 2014; 312(18): 1870-1879.

12. Alfares A, Alfadhel M, Wani T, Alsahli S, Allu-haydan I, Al Mutairi F, et al. A multicenter clinical exome study in unselected cohorts from a consanguineous population of Saudi Arabia demonstrated a high diagnostic yield. Mol Genet Metab. 2017; 121(2): 91-95.

13. Monies D, Abouelhoda M, AlSayed M, Alhassnan Z, Alotaibi M, Kayyali $\mathrm{H}$, et al. The landscape of genetic diseases in Saudi Arabia based on the first 1000 diagnostic panels and exomes. Hum Genet. 2017; 136(8): 921-939.

14. Al-Shamsi A, Hertecant JL, Souid AK, Al-Jasmi FA. Whole exome sequencing diagnosis of inborn errors of metabolism and other disorders in United Arab Emirates. Orphanet J Rare Dis. 2016; 11(1): 94.

15. Fahiminiya S, Almuriekhi M, Nawaz Z, Staffa A, Lepage $\mathrm{P}$, Ali R, et al. Whole exome sequencing unravels disease-causing genes in consanguineous families in Qatar. Clin Genet. 2014; 86(2): 134-141.

16. Makrythanasis P, Nelis M, Santoni FA, Guipponi M, Vannier A, Bena F, et al. Diagnostic exome sequencing to elucidate the genetic basis of likely recessive disorders in consanguineous families. Hum Mutat. 2014; 35(10): 1203-1210.

17. Kalia SS, Adelman K, Bale SJ, Chung WK, Eng C, Evans JP, et al., Recommendations for reporting of secondary findings in clinical exome and genome sequencing, 2016 update (ACMG SF v2.0): A policy statement of the American college of medical genetics and genomics. Genet Med. 2017; 19(2): 249-255.

18. Monies D, Abouelhoda M, Assoum M, Moghrabi N, Rafiullah R, Almontashiri N, et al. Lessons learned from large-scale, first-tier clinical exome sequencing in a highly consanguineous population. Am J Hum Genet. 2019; 104(6), 1182-1201.

19. Charng W-L, Karaca E, Coban Akdemir Z, Gambin T, Atik MM, Gu S, et al. Exome sequencing in mostly consanguineous Arab families with neurologic disease provides a high potential molecular diagnosis rate. BMC Med Genomics. 2016; 9: 42.

20. Sawyer SL, Hartley T, Dyment DA, Beaulieu CL, Schwartzentruber J, Smith A, et al. Utility of wholeexome sequencing for those near the end of the diagnostic odyssey: Time to address gaps in care. Clin Genet. 2016; 89(3): 275-284.

21. Beaulieu CL, Majewski J, Schwartzentruber J, Samules ME, Fernandez BA, Bernier FP, et al. FORGE Canada Consortium: Outcomes of a 2-year national rare-disease gene-discovery project. Am J Hum Genet. 2014; 94(6): 809-817.

22. Mu W, Schiess N, Orthmann-Murphy JL, El-Hattab AW. The utility of whole exome sequencing in diagnosing neurological disorders in adults from a 
highly consanguineous population. J Neurogenet. 2019; 33(1): 21-26.

23. Vissers LELM, van Nimwegen KJM, Schieving JH, Kamsteeg EJ, Kleefstra T, Yntema HG, et al. A clinical utility study of exome sequencing versus conventional genetic testing in pediatric neurology. Genet Med. 2017; 19(9): 1055-1063.

24. Dixon-Salazar TJ, Silhavy JL, Udpa N, Schroth J, Bielas S, schaffer AE, et al. Exome sequencing can improve diagnosis and alter patient management. Sci Trans Med. 2012; 4(138): 138ra78.
25. Zhang X. Exome sequencing greatly expedites the progressive research of Mendelian diseases. Front Med. 2014; 8(1): 42-57.

26. Yavarna T, Al-Dewik N, Al-Mureikhi M, Ali R, AlMesaifri F, Mahmoud L, et al. High diagnostic yield of clinical exome sequencing in Middle Eastern patients with Mendelian disorders. Hum Genet. 2015; 134(9): 967-980. 